\title{
Communication system improvement with control performance based on link quality in wireless sensor actuator networks
}

\author{
Nada N. Tawfeeq, Sawsan D. Mahmood
}

Department of Electrical Engineering, Tikrit University, Iraq

\begin{tabular}{l} 
Article Info \\
\hline Article history: \\
Received Jul 23, 2020 \\
Revised Apr 4, 2021 \\
Accepted Apr 26, 2021 \\
\hline
\end{tabular}

\section{Keywords:}

Communication link quality

Fuzzy logic

Neural networks

WSANs

\begin{abstract}
New communication and networking paradigms started with wireless sensor actuator networks (WSANs) to introduce new applications. One of these is the automatic gain control system (AGC). It will enable a high degree of the decentralized and mobile control. In this study, neural networks (NN) with fuzzy logic (one of the techniques of artificial intelligence (AI)) is used to enhance the control performance depending on the link quality. The $\mathrm{NN}$ and fuzzy inference system (FIS) with Mamdani's method used to build a model reference, adaptive controller, for recompensing for delay time packets losses, and improving the reliability of WSAN. Between $88.62 \%$ and $99.99 \%$, validation data is obtained for the medium and high conditions of operation with the proposed algorithm. Experimental and simulation results show a promising approach.
\end{abstract}

This is an open access article under the CC BY-SA license.

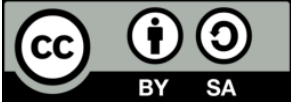

\section{Corresponding Author:}

Nada Nasih Tawfeeq

Department of Electrical Engineering

Tikrit University, Tikrit City, Iraq

Email: nada.nasih@tu.edu.iq

\section{INTRODUCTION}

The communication WSANs system consists of a great number of small devices (sensors and actuators) provided with networking and wireless communication abilities. The importance of WSAN is due to its minimal battery space, tiny equipping space, renewed energy source, fixed buffering capability, small power radio, and minimized power for processing. The functions of sensors are to measure physical quantities such as pressure, temperature, distance [1]. The wireless sensor networks (WSN) are distributed systems utilized to observe and control the implementation of the system. All the nodes of the sensor have been used at random and comprise a network in self-planning technique wirelessly [2]. The stability of the system is affected by the communication link which is a common channel caused by the wave character: the delayed path interference in the transmission process. The WSN link is directing approach leading the changeable communication area [3]. The communication link changed frequently and hence, affects the link quality [4].

The WSANs nodes are used in different topologies to perform certain jobs according to the sensing, processing, and communications performance of these nodes. The WSN consists of a base station or the controller and sensors used for monitoring, managing, and sending the information respectively [5]. The advantages of WN compared to the wired network due to its flexible installations, easy maintenance, mobile operation, and low cost in the wide range of applications like climate observation, army operations, monitoring systems, common security of the public and, healthcare [6]-[7]. Normally, the WN suffers from the data transport issues like dropouts, lagging peripheral accessibility, information errors, unwanted retransmitted data, traffic gridlock, packets lose, latency, path loss, and multipath fading [8].

In the automatic control systems, the issues familiar with wireless links are jitters, delay in time, and packet loss. The delay occurs either among actuators and sensors or among actuators and base-stations. New 
routing algorithm with a fuzzy technique for improving quality of service of WSN to increase in the network operations life, reduce the delay and hence improve the reliability against the failures by reducing the number of packet losses [9].

The optimal dynamic performance of multi-loop control systems by obtains network resources based on the estimation of both control performance and link quality proposed in [10]. Jitter is considered as changeable delays in time among the elements of the WSANs which has a considerable effective on the realtime implementations. This problem might decrease the efficiency of the control system execution and lead to instability [11]. The sensors, actuators, and the other types of equipment are connected to a single controller to improve speed response and operator knowledge of system conditions [12]. In recent years; the WSAN technology plays a vital role in the process-manufactures such as oil refineries steel mills, chemical plants, and infrastructures implementing complex monitoring. The WSANs with standard IEEE 802.15.4 works at small power and cheaply fabricated as presented in [13]. The WSAN configuration is shown in Figure 1.

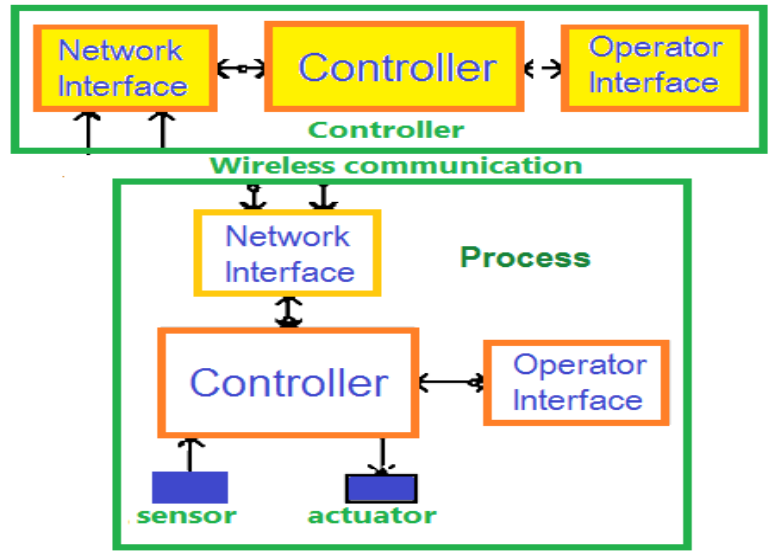

Figure 1. Wireless actuator system configuration

The packet loss rates and the battery supply effects the reliableness of the WSANs. The changeable power transmission, nodes mobility, multi-hop radio interferences, and low radio communication channel power can result from packet losses [14]. In [15], the WSANs' practical aspects of the design and implementation in light control and surveillance have been elaborated. In this study, a neuro-fuzzy logic model for estimation of the link of the WSAN quality is proposed. In order to achieve the objectives, the development of a prototype of a computer-aided system for an intelligent quality of service using a fuzzy logic model is carried out. This study designs a decision support system for the quality of service intelligence system. Fuzzy logic evaluator for the quality of service computing system is designed as well to investigate the system.

\section{RELATED WORKS}

Over the past few years, various researches are done in the communication field in order to study the different sides that are attached to WSANs. In [16], the link quality of the WSAN has been studied by researchers (F. Xia et al.). The method is done by applying the PID algorithm to find the controlling signal from prior information when the output measure is not considered. A new link quality indicator (LQI)-based estimating technique for WSNs is studied, where the packet losses and error packets are taken into consideration. A lot of experiments are carried out on real platforms [17].

The nodes prediction is used for link quality in the future by time series prediction model and the sensor nodes use the spatial correlation of under-water links in [18]. Murray in [19] proposed that the variables of the controller should be adapted in a dynamic manner as stated by link terms. Liu [20] suggested a method of cross-layer design in the wireless control system design and introduced a framework consisting of four layers.

The link quality improvement between nodes using a four-switchable beam antenna in the WSN system in the 2.4 ISM band (2.4-2.485 GHz) is introduced in [21]. Director elements and reflectors achieve reconfigurability of the information in the WSAN. This is activated by PIN diodes [22]. Ibrahim et al. [23] presented an illustration of the reasons that decrease the control system quality and result unstable system. Ngai et al. [24] presented a developed algorithm to decrease the period of waiting for the sensors in the wireless 
sensor actuator networks [24]. In [25] a study on the efforts that have been made to compensate the losses of packets is presented. Methodology for doing the use of a $2.4 \mathrm{GHz}$ network of 18 nodes with a full range of physical link quality indicators is studied in [26]. Minimization of energy with a five-stage neural network and a genetic algorithm is proposed in [27].

\section{COMMUNICATION SYSTEM ARCHITECTURE}

In the WSANs, there are three components designs. The components are actuator, sensor, and basestation (known also as controller). The function of each component has been explained previously. Two designs are used to construct the network, automated and semi-automated. The scheme of the semiautomated which will be utilized to execute the process of the MRANNC is shown in Figure 1. In this architecture; there is no specific controller in the WSANs. The actuator (A) and the controller are directly linked where the measured information of the output from the sensor $(\mathrm{S})$ is received by the controller. When the data is done been processed for some time, a signal is sent to the actuator to get the system carried out depending on a specific algorithm. The error signal is given as (1). The schematic diagram of the proposed communication WSAN system is shown in Figure 2.

$$
e(k)=r(k)-y(k)
$$

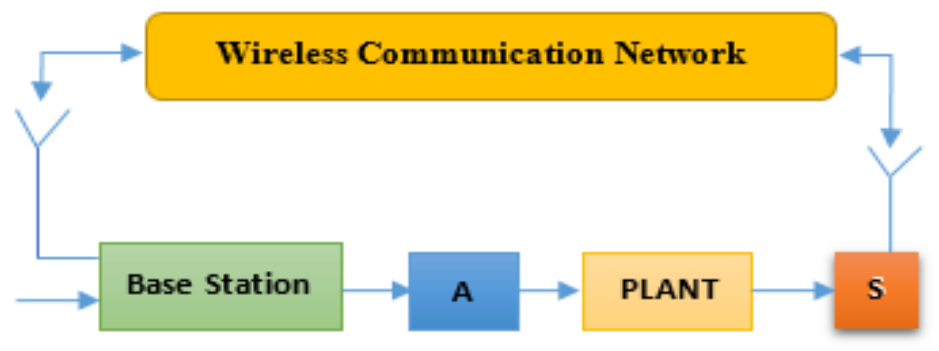

Figure 2. Schematic diagram of communication WSAN system

The measurement of execution of the control system is done by utilizing the performance index (PI). The integral time squared error (ITSE), given by the formula:

$$
I T S E=\int t e^{2}(t) d t
$$

\section{MODEL REFERENCE ADAPTIVE CONTROL}

To control the communication system, the model reference adaptive control is used [28]. It includes two loops, the internal loop which is a simple feedback loop consisting of a regulator and a plant. The regulator variables are brought up to date by the external loop in a manner where the difference between the output of the plant and a pre-specified model output decays. The modification happens when the reference input-signal to the model is varied. The mathematical model of the plant is obtained according to the system identification tool of MATLAB/2018b.

$$
G(s)=\frac{41}{s(0.432 s+1)}
$$

In order to bring the controller variables up to date and estimate the control signal, the adaptive linear (ADALINE) neural network is utilized [29]. The training of the neutral network will save the dynamics of the plant. An estimation of the output of the system is done by the reference model and the neutral network. The graphical scheme of the model adaptive control is shown in Figure 3. The calculation of the control signal is done by the controller $(\mathrm{C})$ and it is decided by the output of neutral network and the model output as given in (4).

$$
u(k)=y_{n}(k)+a_{m 1} y_{p}(k)+a_{m 2} y_{p}(k)+b_{m 1} r(k)+b_{m 2} r(k)
$$


The calculation of output plant can be done as in (5)

$$
\begin{aligned}
& y_{p}(k+1)=a_{m 1} y_{m}(k+1)+a_{m 2} y_{m}(k+1)+ \\
& b_{m 1} r(k+1)+b_{m 2} r(k+1)
\end{aligned}
$$

where $u$ is the control signal., $\mathrm{y}_{\mathrm{m}}$ is the model output, $\mathrm{y}_{\mathrm{p}}$ is the plant output, $\mathrm{y}_{\mathrm{n}}$ is the neural network output, $\mathrm{r}$ is the reference input, am, bm are the model parameters.

This schema is utilized to compensate the losses in packets acknowledging the learning used by NN and the reference model. In order to discuss the link quality of wireless networked control systems, the three matters that will be investigated are:

- Packet loss: In the wireless network, increased chances for data loss exist. Such loss could be the outcome of unstable medium impacts or prolonged range between nodes. Whenever the packet gets lost, the sampling time increased proportionally then it is possible to retransmit of data. The obtained sample is utilized still for calculation and processing.

- Sampling interval: In networked control systems, the Sensors normally work on the sampling process of outputting values in either periodic or aperiodic (event-based) periods. System control is improved when the number of samples is increased in general dynamical systems. When the sampling average gets high, it could lead to overloading in the network. Time delays in the network are introduced due to this reason and thereby, the application of a control system with real-time becomes further difficult number of nodes: One of the reasons for utilizing the technology of wireless communication network is for controlling a number of systems over the exact network. Network overloading could be caused by multiple systems since there could be an increased expectation that greater than one node tries to send information through the exact channel at the exact time.

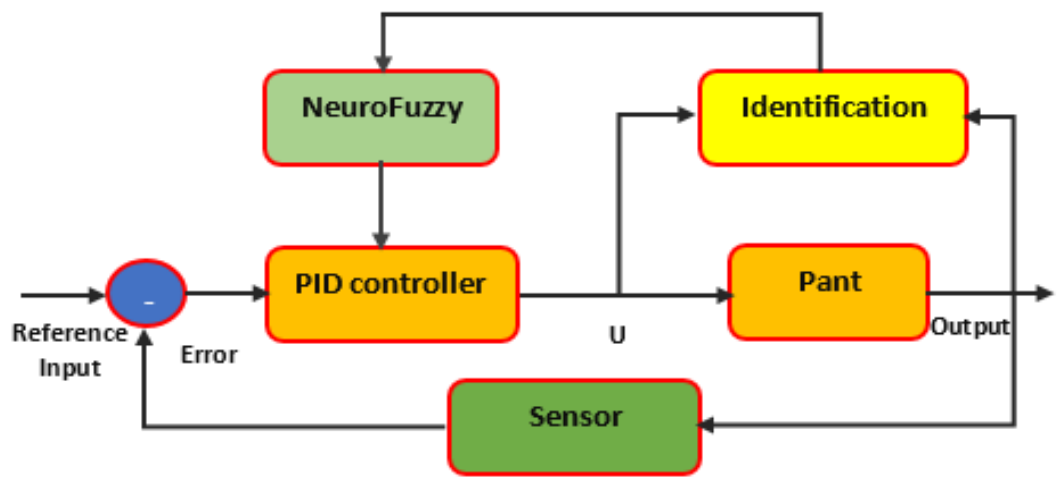

Figure 3. Schematic diagram of the model adaptive control

\section{NEURAL FUZZY APPROACH FOR LINK QUALITY}

In order to improve the quality of service $(\mathrm{QoS})$ in the network, intelligent routing is a significant phenomenon in WSNs for IoT. where, the essential energy for communicating to the IoT based sensor networks is a significant issue to evade enormous packet dropping or packet losses, rapid energy reduction and inequity through the network lead to depletion in the node execution and increment in delays taking into consideration delivering the packets. Subsequently, to improve the execution of the entire network, a maximum requirement is wanted for energy use checking through the implementation of intelligent machine learning techniques to make an efficient routing decision. A number of ways exist in the literature on effective energy routing for WSNs previously. Such approaches should be improved to be suitable with the WSN in IoT medium. Thence, a novel neuro-fuzzy rule based cluster formation and routing protocol to perform effective routing at IoT based WSNs [30]. The proposed algorithm is based on operation with classical WSAN and checks the performance specification with a neuro-fuzzy control system as in Figure 4.

The experimental screen shot of the proposed work is shown in Figure 5. The inputs are divided into three fuzzy sets Low, Medium, and High. 7x7 linguistic variables were used for the outputs, which are negative big (NB), negative medium (NM), negative small (NS), zero (Z), positive small (PS), positive medium (PM), positive big (PB) where it can be listed in Table 1. Thirty rules are used in this work to ensure the best operation. 


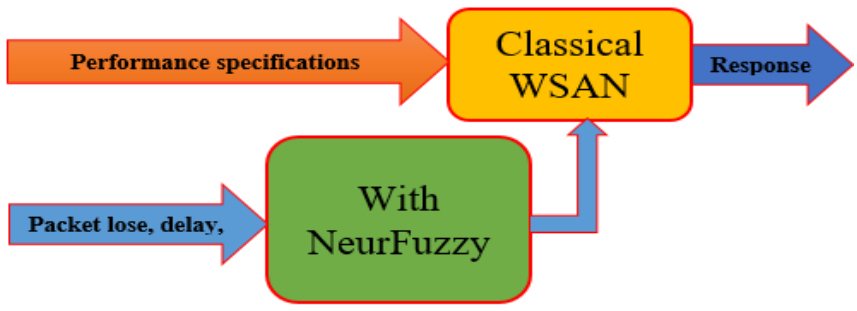

Figure 4. Proposed algorithm schematic diagram

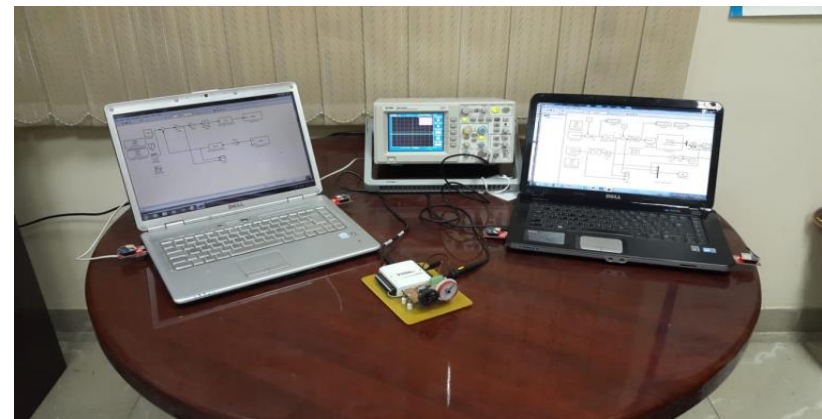

Figure 5. Experimental screen shoot of the work

Table 1. MFs to improve the give tasks

\begin{tabular}{cccccccc}
\hline CE & NB & NM & NS & Z & PS & PM & PB \\
E & & & & & & & \\
\hline NB & NB & NB & NB & NM & NS & NS & Z \\
NM & NB & NM & NM & NM & NS & Z & PS \\
NS & NB & NM & NS & NS & Z & PS & PM \\
Z & NB & NM & NS & Z & PS & PM & PB \\
PS & NM & NS & Z & PS & PS & PM & PB \\
PM & NS & Z & PS & PM & PM & PM & PB \\
PB & Z & PS & PS & PM & PM & PB & PB \\
\hline
\end{tabular}

The membership function (MF) is a triangular membership function. These MFs are suitable for the complex situation and efficient computation. The value of input gets changed to the values of fuzzy by the MFs inputs. Triangular curves depend on three parameters p1, p2, and p3.

$$
\eta(x)=\left[\begin{array}{lc}
0 & \text { if } x<p_{1} \\
x-p_{1} / p_{2}-p_{1} & \text { if } \quad p_{1} \leq x<p_{2} \\
p_{3}-x / p_{3}-p_{2} & \text { if } p_{2} \leq x<p_{3} \\
0 & \text { if } x \geq p_{3}
\end{array}\right.
$$

Where, $\mathrm{p}_{2}$ defines the triangular peak location, while $\mathrm{p}_{1}$ and $\mathrm{p}_{3}$ define the triangular end points.

\section{SIMULATION RESULTS}

The usage of MRANNC to compensate the losses of packets in wireless sensor actuator networks is applied on the next discrete system according to the identification capability of the MATLAB toolbox.

$$
\begin{aligned}
& y(k+1)=-0.0007 y(k)+0.039 y(k-1)+0.853 u(k-1)+ \\
& 0.0406 u(k-2)-0.0011 u(k-3)+0.0046 u(k-4)
\end{aligned}
$$

That system is studied with different step inputs to examine its response. The simulations are implemented by using MATLAB/Simulink and it obvious that the performance and stability have great values by using the proposed neuro-fuzzy control for link quality enhancement.

Communication system improvement with control performance based on link ... (Nada N. Tawfeeq) 
The fuzzy linguistics variables are defined on each input parameters as follows:

- Delay time with three situations low, average, and high,

- $\quad$ Packet loss with three situations low, average, and high.

Low condition is used to describe the highly acceptable QoS Average condition is used to describe the acceptable system response. High condition is used to describe the unacceptable system response. The existence values are selected for input and output parameters as; delay time function $(\mathrm{D}(\mathrm{x}))=[0,400] \mathrm{msec}$, packet loss function $(\operatorname{PKL}(\mathrm{x}))=[0$ to 100$]$ percentage. Where;

$$
x=(D(x), P K L(x)) .
$$

Each linguistic value of fuzzy input and output MF is checked and labeled the degree of the value assigned.

$$
\begin{gathered}
D(x)=\left[\begin{array}{lll}
\text { if } & 70 \leq x>150 & \text { Low delay } \\
\text { if } & 150 \leq x>300 & \begin{array}{l}
\text { Medium delay } \\
\text { if }
\end{array} \\
\text { high delay }
\end{array}\right. \\
\operatorname{PKL}(x)=\left[\begin{array}{lrl}
\text { if } & 10 \leq x>20 & \text { Low delay } \\
\text { if } & 20 \leq x>40 & \text { Medium delay } \\
\text { if } & 40 \leq x>80 & \text { high delay }
\end{array}\right.
\end{gathered}
$$

The fitness function is utilized for the transformation process of the objective function value into a measurement of relative fitness to minimize the lower objective function values. This fitness relationship with objective function can be illustrated as in (10) [31]. The fitness of the system after the objective function application can be shown in Figure 6. The neural network controller for packet loss compensation is shown in Figure 7. The fuzzy logic input variation is illustrated in Figure 8 for stable and smoothly increased input.

$$
\text { Fittness }=\frac{1}{1+\text { ojective function }}
$$

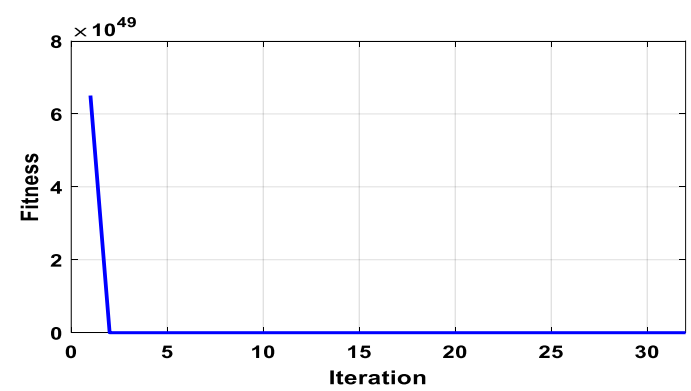

Figure 6. Fitness of the proposed system after 32 iterations

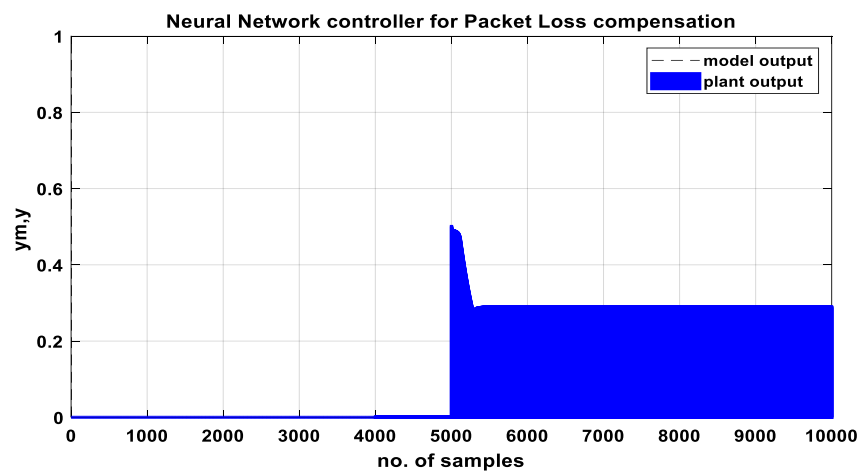

Figure 7. Packet lose compensation with neural network 


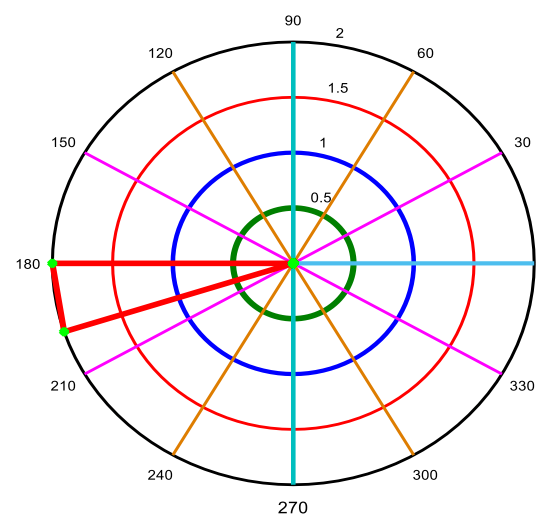

Figure 8. Variation of the Fuzzy logic controller

The response and the control signal of the nonlinear system without the proposed algorithm are shown in Figure 9. In this figure, the response is fast but with a steady-state error which can be canceled or eliminated completely after applying the proposed algorithm. The derivative of the input signal is shown in Figure 10.

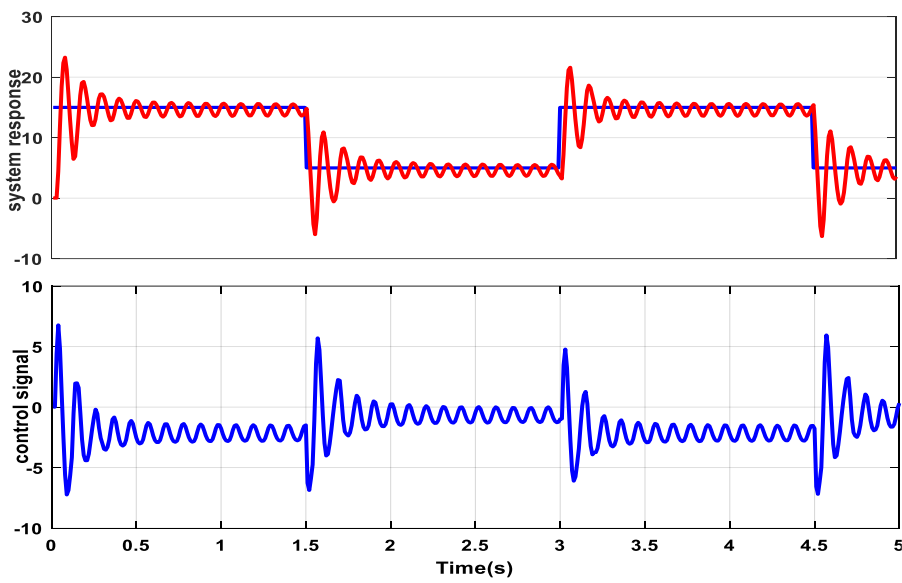

Figure 9. Fuzzy logic of the input system

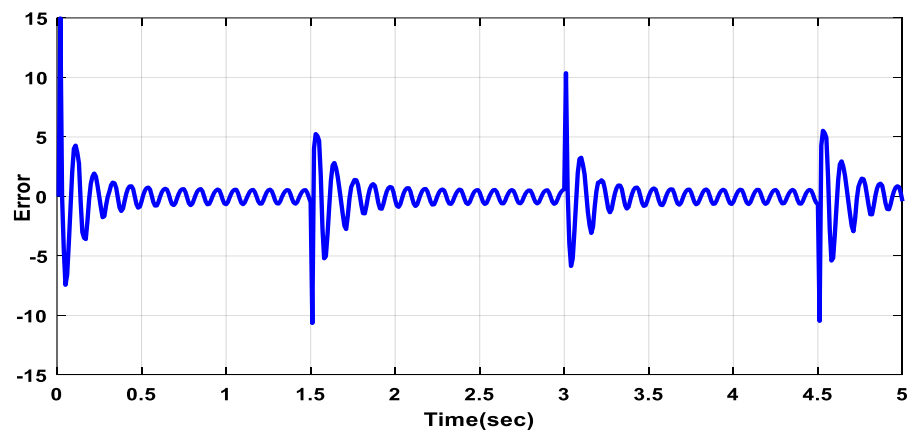

Figure 10. Fuzzy logic

The improvement of the system operation (packet loss, delay time) to medium level is shown in Figure 11. In this system, despite some delay time and the rise time is higher than in Figure 9 but the steadystate error is less. While Figure 12 shows other improvement occurring in the response in the rise time but with steady-state error. 
The validation data of the high and medium levels of operation $(99.9 \%$ and $88.86 \%)$ is shown in Figure 13. These improvements are occurring due to the good selection of the neuro-fuzzy parameters. The complete validation of the neuro-fuzzy of the WSAN is shown in Figure 14. In this figure, fast rise time and zero steady-state error ensure better operation in the system response.

The information transceiver with high efficiency with the proposed algorithm is shown in Figure 15. This ensures efficient QoS of the WSAN system. With the best PID controller gain [Kp=696.1658, $\mathrm{Ki}=111.0314, \mathrm{Kd}=4.9988]$. The Fuzzy logic input-output configuration is shown in Figure 16.

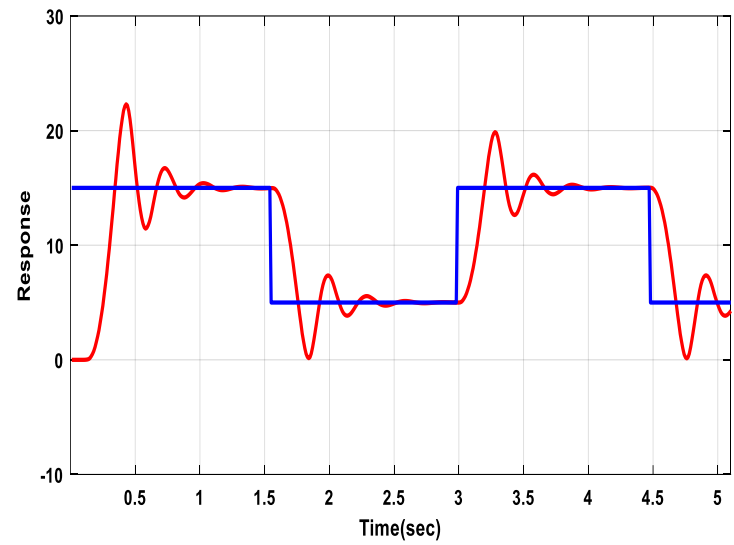

Figure 11. Medium level of the communication link quality

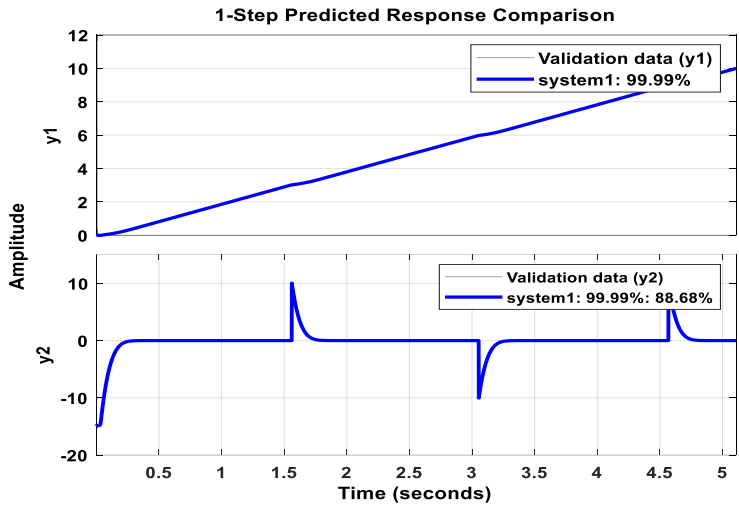

Figure 13. Medium level of the communication link quality

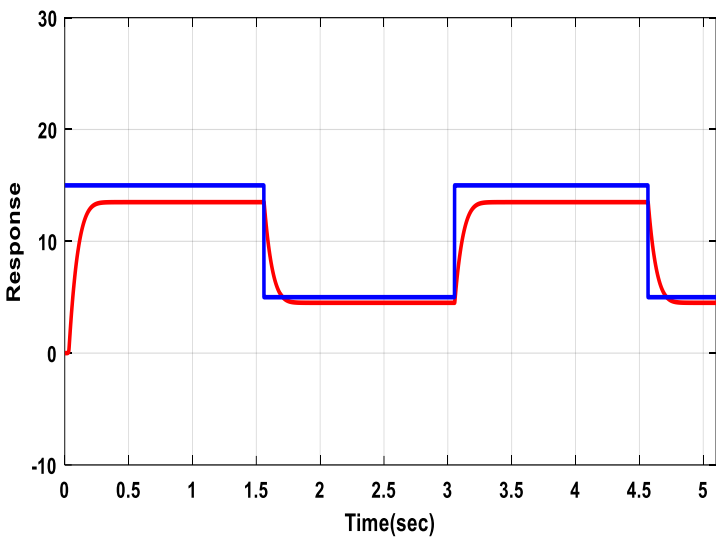

Figure 12. Medium level of the communication link quality

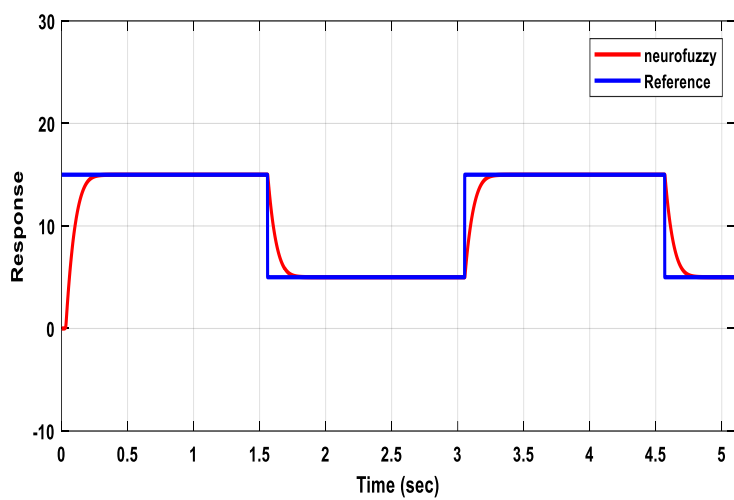

Figure 14. Neuro fuzzy response of the communication system

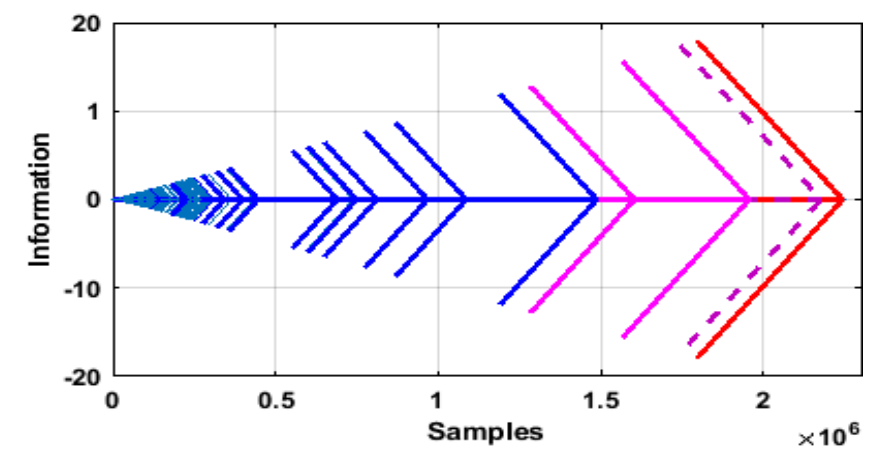

Figure 15. Efficiency of WSAN during the final operation 


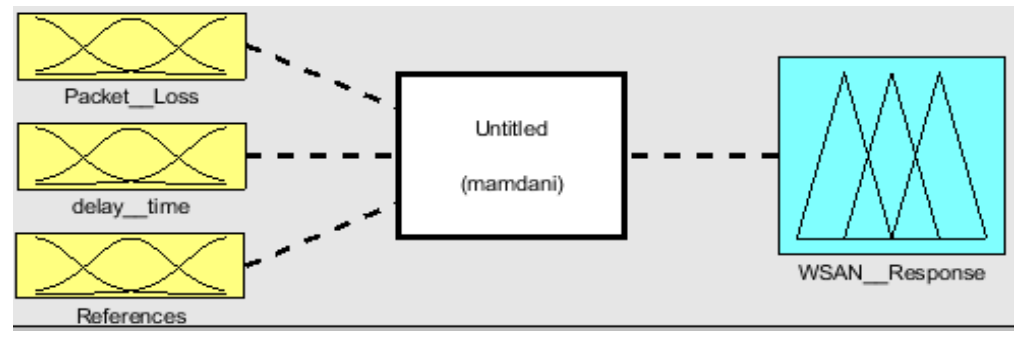

Figure 16. Fuzzy configuration

\section{CONCLUSION}

In this paper, the communication link quality of the wireless sensor actuator networks has been presented and it is applied to a certain control system. As shown in the results, the response of the control system and the best values of the PID controller are obtained. The use of the neural network with Fuzzy logic combined together for the wireless networked control system is studied by constructing a code of MATLAB program to recompense the losses of packets, delay time, and then improve the performing of the networked control system and quality of service (QoS). Though several steps of operation starting with classical WSAN and followed by application of the proposed algorithm, have been reported in this research direction, the application of soft computing in this area provides a better way of handling problems associated can mobility within a wireless mobile network. This work may insure an effective estimation of the implementation of QoS and the network execution. The final concluding remark is that the measured packet loss and delay time are a good sign of the network cases and resource accessibility.

\section{REFERENCES}

[1] K. Y. Lum, J.-S. Chow, and K. H. Yiauw, "Wireless power transfer framework for minirobot based onresonant inductive coupling and impedance matching," International Journal of Power Electronics and Drive System (IJPEDS), vol. 11, no. 1, pp. 317-325, 2020, doi: 10.11591/ijpeds.v11.i1.pp317-325.

[2] H. Sundani, H. Li, V. Devabhaktuni, M. Alam, and P. Bhattacharya, "Wireless sensor network simulators: A survey and comparisons, " International Journal Of Computer Networks (IJCN), vol. 2, no. 5, pp. 249-265, 2011.

[3] S. R. Theodore, "Wireless communications: Principles and practice," SecondEdn. Prentice Hall Press, USA, pp. 72-138, 2011.

[4] A. Woo, T. Tong, and D. Culler, "Taming the underlying challenges of reliable multihop routing in sensor networks," SenSys '03: Proceedings of the 1st international conference on Embedded networked sensor systems, 2003, pp. 14-27, doi: 10.1145/958491.958494.

[5] I. F. Akyildiz and M. Vuran, "Wireless sensor networks," John-Whily\& sons, 2010.

[6] S. Mazumder, "Wireless networking based control," Springer-Verlag New York, 2011, doi: 10.1007/978-1-44197393-1.

[7] R. Elhabyan, W. Shi, and M. St-Hilaire, "Coverage Protocols for Wireless Sensor Networks: Review and Future Directions," Journal of Communications and Network, vol. 21, no. 1, pp. 45-60, 2019, doi: 10.1109/JCN.2019.000005.

[8] A. Nayak and I. Stojmenovic, "Wireless sensor and actuator networks," John-Whiley \& sons, 2010.

[9] R. Abbasi, A. M. Bidgoli, and M. A. Dezfoli, "A New Fuzzy Algorithm for Improving Quality of Service in Real Time Wireless Sensor Networks," International Journal Of Advanced Smart Sensor Network Systems (IJASSN), vol. 2, no. 2, pp. 1-14, 2012, doi: 10.5121/ijassn.2012.2201.

[10] Y. Ma et al., "Optimal Dynamic Scheduling of Wireless Networked Control Systems," Proceedings of the 10th ACM/IEEE International Conference on Cyber-Physical Systems, Montreal, Quebec, Canada, 2009, pp. 77-86, doi: 10.1145/3302509.3311040.

[11] "Control System Architecture-Instrumentation Tools," 2019. [Online]. Available: https://instrumentation tools.com/control system architecture/2019.

[12] J. Shi and M. Sha, "Parameter Self-Configuration and Self-Adaptation in Industrial Wireless Sensor-Actuator Networks," IEEE Conference on Computer Communications, France, 2019, pp. 658-666, doi: 10.1109/INFOCOM.2019.8737467.

[13] T. Melodia, D. Pompili, and I. F. Akyldiz, "Handling mobility in wireless sensor and actuator networks," IEEE Transaction on mobile computing, vol. 9, no. 2, pp. 160-173, 2010, doi: 10.1109/TMC.2009.102.

[14] S. Li, "Wireless sensor actuatornetworks for light monitoring and control applications," CCNC 2006. $20063 \mathrm{rd}$ IEEE Consumer Communications and Networking Conference, vol. 2, 2006, pp. 974-978, 2006, doi: 10.1109/CCNC.2006.1593183.

[15] J. Luo, L. Yu, D. Zhang, Z. Xia and W. Chen, "A New Link Quality Estimation Mechanism Based on LQI in WSN," Information Technology Journal, vol. 12, pp. 1626-1631, 2013, doi: 10.3923/itj.2013.1626.1631.

[16] F. Xia, Y.-C. Tian, Y. Li, and Y. Sun, "Wireless sensor/actuator networks for mobile control," Sensors, vol. 7, no. 10, pp. 2157-2173, 2007, doi: 10.3390/s7102157. 
[17] L. Wang, F. Xiao, and C. Huang, "Adaptive Topology Control with Link Quality Prediction for Underwater Sensor Networks," Adhoc\& Sensor Wireless Networks, vol. 43, no. 3/4, pp. 179-212, 2019.

[18] M. A. Ibrahim, A. Kh, and N. Sultan, "Optimal PID controller of a brushless dc motor using genetic algorithm," International journal of power electronic and drive systems (IJPEDS), vol. 10, no. 2, pp. 822-830, 2019, doi: 10.11591/ijpeds.v10.i2.822-830.

[19] Y. Mostofi, and R. Murray, "Effect of time varying fading channels on the control performance of mobile sensor node," 2004 First Annual IEEE Communications Society Conference on Sensor and Ad Hoc Communications and Networks, 2004. IEEE SECON 2004., 2004, pp. 317-324, doi: 10.1109/SAHCN.2004.1381932.

[20] X. Liu and A. Goldsmith "Wireless network design for distributed control," 2004 43rd IEEE Conference on Decision and Control (CDC) (IEEE Cat. No.04CH37601), 2004, pp. 2823-2829, 2004, doi: 10.1109/CDC.2004.1428892.

[21] A. Ayan, A. Diallo, P. Le Thuc, and R. Staraj, "Directive and Reconfigurable Antenna for Wireless Sensor Network to Improve Link Quality between Nodes," IEEE Sensors Applications Symposium (SAS), France, 2019, pp. 1-5, doi: 10.1109/SAS.2019.8706061.

[22] D. Rojas and J. Barrett, "Link quality evaluation of a wireless sensor network in metal marine environments," Wireless Networks. vol. 25, pp. 1253-1271, 2019, doi: 10.1007/s11276-018-1726-z.

[23] I. Alhamrouni, M. Iskandar, M. Salem, L. Awalin, A. Jusoh, T. Sutikno, "Application of inductive coupling for wireless power transfer," international journal of power electronic and drive systems (IJPEDS), vol. 11, no. 3, pp. 1009-1116, 2020, doi: 10.11591/ijpeds.v11.i3.pp1109-1116.

[24] E. Ngai, J. Liu, and M. R. Lyu, "Delay-minimized route design for wireless sensor-actuator networks," 2007 IEEE Wireless Communications and Networking Conference, 2007, pp 3675-3680, doi: 10.1109/WCNC.2007.673.

[25] J. Haspanha, P. Naghshtabrizi, and Y. Xu, "Survey of recent results in networked control systems," Proceedings of the IEEE, vol. 95, no. 1, pp. 132-162, 2007, doi: 10.1109/JPROC.2006.887288.

[26] B. Prakash, S. Jayashri, and T. S. Karthik, "A hybrid genetic artificial neural network (G-ANN) algorithm for optimization of energy component in a wireless mesh network toward green computing," Soft Computing, vol. 23, pp 2789-2798, 2019, doi: 10.1007/s00500-019-03789-8.

[27] V. P. Tran, F. Santoso, M. A. Garratt, and S. G. Anavatti, "Distributed Artificial Neural Networks-Based Adaptive Strictly Negative Imaginary Formation Controller for Unmanned Aerial Vehicles in Time-Varying Environments," IEEE Transactions on Industrial Informatics, vol. 17, no. 6, pp. 3910-3919, 2020, doi: 10.1109/TII.2020.3004600.

[28] M. V. B. Sergey and G. Emelyanov, "A nonlinear method of learning neuro-fuzzy models for dynamic control systems," Applied Soft Computing, vol. 88, 2020, Art. no. 106030, doi: 10.1016/j.asoc.2019.106030.

[29] K. Thangaramy, K. Kulothungan, R. Logambigai, M. Selvi, S. Ganapathy, and A. Kannan, "Energy aware cluster and neuro-fuzzy based routing algorithm for wireless sensor networks in IoT," Computer Networks, vol. 151, no. 14, pp. 211-223, 2019, doi: 10.1016/j.comnet.2019.01.024.

[30] U. A. Umoh and D. Asuquo, "Fuzzy Logic Based Quality of Service Evaluation in Multimedia Transmission over AD Hoc Wireless Network," International Journal of Computational Intelligence and Applications, vol. 16, no. 14, pp. 1-22, 2018, Art. no. 1750023, doi: 10.1142/S1469026817500237.

[31] P. Kawka and A. Alleyne, "Stability and feedback control of wireless networked systems," Proceedings of the 2005, American Control Conference, Portland, OR, USA, 2005, pp. 2953-2959, 2005, doi: 10.1109/ACC.2005.1470423. 